\title{
Properly edge-coloured subgraphs in colourings of bounded degree
}

\author{
Klas Markström \\ Institutionen för matematik och matematisk statistik \\ Umeå universitet \\ Andrew Thomason \\ DPMMS, Centre for Mathematical Sciences \\ Wilberforce Road, Cambridge CB3 0WB \\ Peter Wagner* \\ Department Mathematik, Universität Hamburg \\ Bundesstr. 55, 20146 Hamburg
}

19th September 2009

\begin{abstract}
The smallest $n$ such that every colouring of the edges of $K_{n}$ must contain a monochromatic star $K_{1, s+1}$ or a properly edge-coloured $K_{t}$ is denoted by $f(s, t)$. Its existence is guaranteed by the Erdős-Rado Canonical Ramsey theorem and its value for large $t$ was discussed by Alon, Jiang, Miller and Pritikin [1].

In this note we primarily consider small values of $t$. We give the exact value of $f(s, 3)$ for all $s \geq 1$ and the exact value of $f(2,4)$, as well as reducing the known upper bounds for $f(s, 4)$ and $f(s, t)$ in general.
\end{abstract}

\section{Properly edge-coloured subgraphs}

Given graphs $G$ and $H$, let $\mathcal{R}(G, H)$ be the smallest $n$ such that every colouring of the edges of $K_{n}$ must contain a monochromatic $G$ or a rainbow $H$ (meaning a copy of $H$ in which no two edges have the same colour). The Erdös-Rado Canonical Ramsey theorem [3] implies that $\mathcal{R}(G, H)$ exists if and only if either $G$ is a star or $H$ is acyclic.

A systematic study of $\mathcal{R}(G, H)$ was begun by Jamison, Jiang and Ling [7] and by Chen, Schelp and Wei [2]. However certain cases were already in the literature - for instance, the case when $H$ is the star $K_{1, k+1}$ is the $k$-local Ramsey number of $G$ (see Gyárfás, Lehel, Schelp and Tuza [5]). When $H$ is a path, the case when $G$ is also a path was highlighted in [7] and studied in [15],

\footnotetext{
${ }^{*}$ Research funded by Trinity College, University of Cambridge.
} 
and in [14] it was shown that if $H$ is a path of length $\ell \leq 5$ (the length being the number of edges) then $\mathcal{R}(G, H)$ is effectively determined by the $(\ell-1)$-colour Ramsey number for $G$. On the other hand, Alon, Jiang, Miller and Pritikin [1] concentrated on the case when $G$ is a star. They looked especially at the case when $G$ is complete, which was implicit in the work of Lefmann and Rödl [9] on Canonical Ramsey numbers. This is the focus of the present note also.

Following [1], for $s \geq 1$ and $t \geq 2$ we define $g(s, t)=\mathcal{R}\left(K_{1, s+1}, K_{t}\right)$, and we define $f(s, t)$ to be the smallest $n$ such that every colouring of the edges of $K_{n}$ must contain a monochromatic $K_{1, s+1}$ or a properly edge-coloured $K_{t}$ (meaning a $K_{t}$ within which no two incident edges have the same colour). It follows from the definitions that $f(s, t) \leq g(s, t)$. Plainly $f(s, 2)=g(s, 2)=2$ and $f(1, t)=t$. Henceforth we shall assume that $s \geq 2$ and $t \geq 3$.

It is proved in [1] that $g(s, t)=\Theta\left(s t^{3} \log t\right)$. The function $f(s, t)$ is also estimated well, though not quite so precisely; it is shown that $c s t^{2} / \log t \leq$ $f(s, t) \leq 4 s t^{2}$ for some constant $c$. The small gap here prompted us to look more closely at $f(s, t)$, beginning with small values of $t$.

Clearly $f(s, 3)=g(s, 3)$. We give the precise value of $f(s, 3)$ in the next section. In $\S 3$ we show that $f(2,4)=10$ and give bounds on $f(s, 4)$. Finally in $\S 4$ we improve slightly the upper bound for $f(s, t)$.

\section{Rainbow triangles}

Take a 2-coloured $K_{5}$ consisting of a red and a blue 5-cycle. Substitute a green complete graph into each vertex, so that the edges between two green graphs are the same colour as the edge between the original two vertices. If each green graph has $k$ vertices we get a $K_{5 k}$ with no monochromatic $K_{1,2 k+1}$. If one green graph has $k+1$ vertices and the others have $k$ then we get a $K_{5 k+1}$ with no monochromatic $K_{1,2 k+2}$. In neither case is there a rainbow $K_{3}$. These colourings establish lower bounds on $f(s, 3)$ which are tight.

Theorem $1 f(s, 3)=5 s / 2+1$ if $s$ is even and $f(s, 3)=5 s / 2-1 / 2$ if $s$ is odd.

Proof. A direct proof can be found in [16] but a shorter proof follows Gyárfás and Simonyi [6], who called colourings of the edges of $K_{n}$ with no rainbow $K_{3}$ Gallai colourings on the basis of Gallai's work [4]. They gave a simple argument that every Gallai colouring with $n \geq 2$ consists of substituting Gallai colourings into the vertices of a 2-coloured complete graph on at least two vertices. Let $K_{n}$ be Gallai coloured and let there be $\ell$ vertices in the 2 -coloured complete graph (coloured red and blue, say). As noted in [6], if $\ell \leq 4$ then some vertex of $K_{n}$ has degree at least $n / 2$ in red or blue, and if $\ell \geq 5$ then a vertex in the smallest block has degree at least $2 n / 5$ in red or blue, which already proves that $f(s, 3) \leq 5 s / 2+1$ if $s$ is even (this is Theorem 3.1 of $[6]$ ).

So let $s=2 k+1$ and $n=5 k+2$, and suppose $K_{n}$ is Gallai coloured with no monochromatic $K_{1,2 k+2}$. There are $\ell \geq 5$ blocks, and each vertex is joined to at most $2(2 k+1)=4 k+2$ vertices in other blocks. Thus each block has size at least $k$. Therefore $\ell \leq 7$. If $\ell=7$ then $k=1$ and every block has size one. But each vertex of the 2-coloured $K_{7}$ has degree at most three in red and blue, which is impossible: thus $\ell \leq 6$. If $\ell=6$ then either $k=2$ or $k=1$. The first is impossible, for it implies every block has size 2 , and then some block is joined to 3 others by red, say, giving a monochromatic $K_{1,6}$. So $k=1$ and there are 
five blocks of size 1 and one, $B$ say, of size 2 . The five vertices not in $B$ are each joined to three vertices by red (and by blue), and the two vertices in $B$ have the same degree in red, which is impossible. So $\ell=5$.

So there are five blocks, three or four of which have size $k$. If a block has size $k$ then the remaining four blocks must be partitionable into two sets of size $2 k+1$. So if there are four blocks size $k$, and one of size $k+2$, then $k=1$. But this is impossible, because the block of size 3 must be joined to at least one singleton, $x$, by red and to another, $y$, by blue; by symmetry we may assume $x y$ is red, and then $x$ is the centre of a red $K_{1,4}$. Hence there are three blocks of size $k$ and two of size $k+1$. Each small block must be joined to the two large ones by edges of different colours. So there must be two small blocks both joined to one large block by red and to the other large block by blue. By symmetry we may assume the large blocks are joined to each other by red; but then each vertex in a large block is at the centre of a red $K_{1,3 k+1}$, a contradiction.

\section{Properly edge-coloured $K_{4} \mathrm{~S}$}

In this section we consider $f(s, 4)$ for $s \geq 2$. We begin with lower bounds.

Lemma $2 f(2,4)>9$.

Proof. Label the vertices of $K_{9} v_{0}, v_{1}, \ldots, v_{8}$. Let $C_{i}, 0 \leq i \leq 8$, be the 4 cycle with edges $v_{i} v_{i-1}, v_{i-1} v_{i+1}, v_{i+1} v_{i+4}, v_{i+4} v_{i}$, with suffixes taken modulo 9 . Since each cycle contains exactly one edge whose suffixes differ by $j, 1 \leq j \leq 4$, it follows that the nine 4-cycles form a (rotationally symmetric) partition of the 36 edges of $K_{9}$. Colour the edges of $C_{i}$ with colour $i$. Then we have a colouring with no monochromatic $K_{1,3}$. Suppose there is a properly edge-coloured $K_{4}$. If no two vertices have consecutive suffixes then we may assume (by symmetry) that they are $v_{0}, v_{2}, v_{4}, v_{6}$. But $v_{4} v_{6}$ and $v_{6} v_{0}$ both have colour 5 . So we may assume that $v_{0}$ and $v_{1}$ are in the $K_{4}$. Since $v_{0} v_{1}$ has colour 1 , neither $v_{2}$ nor $v_{5}$ is in the $K_{4}$. Neither $v_{4}$ nor $v_{8}$ can be in the $K_{4}$ else they would meet two edges of colour 0 . The three remaining possibilities for $V\left(K_{4}\right)$, namely $\left\{v_{0}, v_{1}, v_{3}, v_{6}\right\},\left\{v_{0}, v_{1}, v_{3}, v_{7}\right\}$ and $\left\{v_{0}, v_{1}, v_{6}, v_{7}\right\}$, are ruled out by colours 2,8 and 6 respectively. This completes the proof.

It is interesting to observe that this construction uses nine colours on only four edges each; intuition might suggest that employing few colours, perhaps only four (each used on a 2-factor), would be a good idea, but this is not the case. The construction has implications for larger $s$ too.

Lemma $3 f(s, 4)>9 s / 2$ for even $s$ and $f(s, 4)>9 s / 2-3 / 2$ for odd $s$.

Proof. The lower bounds come from a natural blowup method also used in [1]. If $s=2 k$, take the colouring of $K_{9}$ given by Lemma 2 and substitute $K_{k} \mathrm{~s}$ into each vertex, coloured with a new colour, in the manner of $\S 2$. This gives a colouring of $K_{9 k}$ with no monochromatic $K_{1,2 k+1}$. If $s=2 k+1$, we substitute $K_{k+1}$ into $v_{0}, v_{3}$ and $v_{6}$ and substitute $K_{k}$ s into the other six vertices. Since no vertex of $K_{9}$ is joined to two of $\left\{v_{0}, v_{3}, v_{6}\right\}$ by the same colour, the colouring of $K_{9 k+3}$ contains no monochromatic $K_{1,2 k+2}$. 
Any $K_{4}$ in the $K_{9 k}$ or $K_{9 k+3}$ that has its vertices in pairwise distinct substituted complete graphs is properly edge-coloured because it is coloured in the same way as a $K_{4}$ in the coloured $K_{9}$. Any other $K_{4}$ either has more than two vertices in one complete graph or it has two in one complete graph and at least one in another. In neither case is the $K_{4}$ properly edge-coloured, completing the proof.

Upper bounds for $f(s, 4)$, and for $f(s, t)$ in general, make use of estimates of the number of monochromatic paths of length two. The following bound applies generally.

Lemma 4 Let $K_{n}$ be edge-coloured with no monochromatic $K_{1, s+1}$. Then there are at most $(s-1)\left(\begin{array}{l}n \\ 2\end{array}\right)$ monochromatic paths of length two.

Proof. Each monochromatic path of length two contains two edges. Each of the $\left(\begin{array}{l}n \\ 2\end{array}\right)$ edges of $K_{n}$ lies in at most $2(s-1)$ such paths.

The upper bound $f(s, 4) \leq 64 s$ was given in [1]. One way of improving this is by arguing as follows. Let $n=f(s, 4)-1$ and let $K_{n}$ be coloured with no monochromatic $K_{1, s+1}$ and no properly edge-coloured $K_{4}$. By Lemma 4 we can find two vertices $v$ and $w$ such that there are at most $s-1$ vertices $u$ for which the path $v u w$ is monochromatic. Let $X$ be the set of vertices $x$ such that $\{x, v, w\}$ spans no monochromatic path (i.e. spans a rainbow triangle). Since there is no monochromatic $K_{1, s+1}$ we have $|X| \geq(n-2)-3(s-1)$. Let $F$ be the graph on vertex set $X$ consisting of edges $x y$ for which at least one of $x v y$ and $x w y$ is monochromatic. Each vertex of $X$ has degree at most $2(s-1)$ in $F$, and so $F$ has at most $|X|(s-1)$ edges. Given $x \in X$, let $M_{x}$ be the set of edges $x y$ with $y \in X$ for which at one of $v x y$ and $w x y$ is monochromatic. Then $\left|M_{x}\right| \leq 2(s-1)$. Observe now that, for any edge $x y$ with $x, y \in X$, either $x y$ is an edge of $F$ or $x y \in M_{x} \cup M_{y}$, because otherwise $\{v, w, x, y\}$ spans a properly edge-coloured $K_{4}$. Thus $\left(\begin{array}{c}|X| \\ 2\end{array}\right) \leq 3|X|(s-1)$ or $|X| \leq 6 s-5$. Hence $f(s, 4)=n+1 \leq|X|+3 s \leq 9 s-5$. This upper bound is less than twice the lower bound given by Lemma 3 .

An alternative approach to bounding $f(s, 4)$ is by associating a 3 -uniform hypergraph $H$ with each edge-colouring of $K_{n}$. The vertex set of $H$ is the same as that of $K_{n}$, and the edges of $H$ are those triples not spanning a rainbow triangle - that is, those containing a monochromatic path of length two. An independent set of size 4 in $H$ corresponds exactly to a properly edge-coloured $K_{4}$ in $K_{n}$. The number of edges in $H$ is bounded above (say, by Lemma 4 ). If $n$ is large enough then the number of edges in $H$ will be less than the number needed to prevent the occurrence of an independent 4-set. In this way we obtain an upper bound on $f(s, 4)$. This argument is the basis of the bounds in [1] though by applying it more carefully we get somewhat reduced bounds. (Of course, in [1] there was no attempt to optimize the constants.)

Let $m_{4}(n)$ be the minimum number of edges in a 3-uniform hypergraph of order $n$ with no independent 4-set, and let $\mu_{4}(n)=m_{4}(n)\left(\begin{array}{l}n \\ 3\end{array}\right)^{-1}$ be the minimum density. It is easily shown that $\mu_{4}(n)$ is an increasing function of $n$.

Most of the inequalities $m_{4}(4)>0, m_{4}(5)>2, m_{4}(6)>5, m_{4}(7)>11$, $m_{4}(8)>18, m_{4}(9)>28$ and $m_{4}(10)>40$ are easily established by induction; for example, a hypergraph of order 9 having 28 edges has a vertex of degree 
at least 10, whose removal leaves a hypergraph with at most 18 edges, and so $m_{4}(8)>18$ implies $m_{4}(9)>28$. The only inequality that does not follow this way is $m_{4}(7)>11$ : the truth of this can be established by a short direct argument (see [16]) or by computer.

We make these remarks because the inequality $m_{4}(10)>40$ is what we need for the next theorem. In fact, the identities $m_{4}(4)=1, m_{4}(5)=3, m_{4}(6)=6$, $m_{4}(7)=12, m_{4}(8)=20$ and $m_{4}(9)=30$ are reported by Katona, Nemetz and Simonovits [8], $m_{4}(10)=45$ by Stanton and Bate [13] and $m_{4}(11)=63$, $m_{4}(12)=84$ and $m_{4}(13)=112$ by Radziszowski and Zou [12, p. 187]. These figures have been verified and extended by one of us $[10,11]$.

We can now evaluate $f(2,4)$ precisely.

Theorem $5 f(2,4)=10$.

Proof. Lemma 2 shows $f(2,4) \geq 10$. Suppose now that we have a $K_{10}$ edgecoloured with no monochromatic $K_{1,3}$. There are at most four monochromatic paths of length two centred at each vertex, and so the associated 3-uniform hypergraph $H$ of order 10 has at most 40 edges. Since $m_{4}(10)>40$ this means $H$ has an independent 4 -set, so the $K_{10}$ has a properly edge-coloured $K_{4}$. Hence $f(2,4) \leq 10$.

The next theorem provides a simple upper bound which improves on our previous $9 s-5$ and so is closer to the lower bound of Lemma 3 .

Theorem $6 f(s, 4) \leq 15 s / 2$.

Proof. Let $n=\lfloor 15 s / 2\rfloor$ and suppose that $K_{n}$ is edge-coloured without a monochromatic $K_{1, s+1}$ and without a properly edge-coloured $K_{4}$. Of the $n-1$ edges at a vertex, at most $s$ are the same colour, and so the number of monochromatic paths of length two centred at that vertex is at most $7\left(\begin{array}{l}s \\ 2\end{array}\right)+\left(\begin{array}{c}r-1 \\ 2\end{array}\right)$ where $7 s+r=n$ and $r=\lfloor s / 2\rfloor \geq 1$. So the number of edges in the associated 3uniform hypergraph $H$ is at most $n\left(7\left(\begin{array}{c}s \\ 2\end{array}\right)+\left(\begin{array}{c}r-1 \\ 2\end{array}\right)\right)$. Since $H$ has no independent 4-set the density of $H$ is at least $\mu_{4}(n)$. Because $n>13$ we have $\mu_{4}(n) \geq$ $\mu_{4}(13)=112\left(\begin{array}{c}13 \\ 3\end{array}\right)^{-1}=56 / 143>7 / 18$, using the data mentioned earlier. Hence the inequality

$n\left(7\left(\begin{array}{l}s \\ 2\end{array}\right)+\left(\begin{array}{c}r-1 \\ 2\end{array}\right)\right) \geq \frac{7}{18}\left(\begin{array}{l}n \\ 3\end{array}\right), \quad$ that is, $7\left(\begin{array}{l}s \\ 2\end{array}\right)+\left(\begin{array}{c}r-1 \\ 2\end{array}\right) \geq \frac{7}{54}\left(\begin{array}{c}n-1 \\ 2\end{array}\right)$

holds. Writing $r=(s-\delta) / 2$, where $\delta=0$ or 1 , we have $s=2 r+\delta$ and $n=15 r+7 \delta$. After substituting these expressions for $s$ and $n$ into the inequality, multiplying by 108 and rearranging, remembering that $\delta^{2}=\delta$, we obtain $-9 r^{2}+$ $42 r \delta-603 r+94-196 \delta \geq 0$. Therefore $-9 r^{2}-561 r+94 \geq 0$. But this inequality is not satisfied for any $r \geq 1$, a contradiction which completes the proof.

The inequality $m_{4}(19) \geq 574$ is given in $[10,11]$ and this can be used to improve Theorem 6 to $f(s, 4) \leq 7.304 s$. Note that using the limit $\mu_{4}(n) \rightarrow 4 / 9$, conjectured by Turán, in the previous argument would bring the upper bound only just below $13 s / 2$ for large $s$. 


\section{A general bound}

We conclude by observing a slight improvement on the bound $f(s, t) \leq 4 s t^{2}$ of [1]. Given an edge-colouring of $K_{n}$ with no monochromatic $K_{1, s+1}$ or properly edge-coloured $K_{t}$, we associate with it a 3 -uniform hypergraph as in the previous section. The upper bound on the number of edges of $H$ given by Lemma 4 applies. But $H$ has no independent $t$-set. Write $m_{t}(n)$ and $\mu_{t}(n)$ for the minimum number of edges and minimum density, respectively, of a 3uniform hypergraph of order $n$ with no independent $t$-set. As before, $\mu_{t}(n)$ increases with $n$. In [1] the bound $m_{t}(2 t)>t$ was used. Writing $\alpha=t-1$, Sidorenko [12, equation (33) p. 192] states the values $m_{t}((9 \alpha+1) / 4)=3 \alpha+3$, $m_{t}(9 \alpha / 4)=3 \alpha, m_{t}((9 \alpha-1) / 4)=3 \alpha+1$ and $m_{t}((9 \alpha+2) / 4)=3 \alpha+4$, valid for $t \equiv 0,1,2$ and $3(\bmod 4)$ respectively. In each case, it is readily verified that the density $\mu_{t}(n)=m_{t}(n)\left(\begin{array}{l}n \\ 3\end{array}\right)^{-1}$ is at least $128 / 81 \alpha^{2}$. Also, $n<3 \alpha$ in each case.

Theorem $7 f(s, t) \leq 3^{5} 2^{-7}(s-1)(t-1)^{2}+3<1.899(s-1)(t-1)^{2}+3$.

Proof. Theorem 1 means we can assume $t \geq 4$. Let $n=f(s, t)-1$ and let $K_{n}$ be edge-coloured with no monochromatic $K_{1, s+1}$ or properly edge-coloured $K_{t}$. Let $H$ be the associated 3-uniform hypergraph. By Lemma $4, H$ has at most $(s-1)\left(\begin{array}{l}n \\ 2\end{array}\right)$ edges, so it has density at most $3(s-1) /(n-2)$. Since $H$ has no independent $t$-set, its density is at least $\mu_{t}(n)$. Either $n<3 t$, in which case the theorem is true anyway, or $n \geq 3 t$ and so, by the remarks preceding the theorem, $\mu_{t}(n) \geq 128 / 81(t-1)^{2}$. The theorem follows.

\section{References}

[1] N. Alon, T. Jiang, Z. Miller and D. Pritikin, Properly colored subgraphs and rainbow subgraphs in edge-colorings with local constraints, Random Structures and Algorithms 23 (2003), 409-433.

[2] G. Chen, R. Schelp and B. Wei, Monochromatic-rainbow Ramsey numbers, 14th Cumberland Conference Abstracts, May 2001. URL http://www.msci.memphis. edu/〜pbalistr/Abstracts.html.

[3] P. Erdős and R. Rado, A combinatorial theorem, J. London Math. Soc. 25 (1950) 249-255.

[4] T. Gallai, Transitiv orientierbare Graphen, Acta Math. Acad. Sci. Hungar 18 (1967) 25-66.

[5] A. Gyárfás, J. Lehel, R.H. Schelp and Zs. Tuza, Ramsey numbers for local colorings, Graphs Combin. 3 (1987) 267-277.

[6] A. Gyárfás and G. Simonyi, Edge colorings of complete graphs without tricolored triangles, J. Graph Theory 46 (2004) 211-216.

[7] R.E. Jamison, T. Jiang and A.C.H. Ling, Constrained Ramsey numbers of graphs, J. Graph Theory 42 (2003) 1-16. 
[8] Gy. Katona, T. Nemetz and M. Simonovits, On a problem of Turán in the theory of graphs, (in Hungarian) Mat. Lapok 15 (1964) 228-238.

[9] H. Lefmann and V. Rödl, On Erdős-Rado numbers, Combinatorica 15 (1995), 85-104.

[10] K. Markström, Turan graphs for 3-uniform complete graphs of small order, Manuscript.

[11] K. Markström. A web archive of turan graphs. URL http://abel.math.umu.se/ ${ }^{k l a s m / D a t a / h y p e r g r a p h s / t u r a n h y p e r g r a p h s . h t m l . ~}$

[12] A. Sidorenko, What we know and what we do not know about Turan numbers, Graphs and Combinatorics 11 (1995), 179-199.

[13] R.G. Stanton and J.A. Bate, A computer search for B-coverings, Lecture Notes in Math. 829, Springer, Berlin (1980), 37-50.

[14] A. Thomason and P. Wagner, Complete graphs with no rainbow path, $J$. Graph Theory 54 (2006), 261-266.

[15] P. Wagner, An upper bound for constrained Ramsey numbers, Combinatorics, Probability and Computing 15 (2006), 619-626.

[16] P. Wagner, Ph.D. thesis, University of Cambridge (2006). 